\title{
Factors associated with thoracic spinal cord injury, lesion level and rotator cuff disorders
}

\author{
KA Sinnott*,1, P Milburn ${ }^{2}$ and H McNaughton ${ }^{1}$ \\ ${ }^{1}$ Rehabilitation Teaching and Research Unit, Wellington School of Medicine, University of Otago, Dunedin, \\ New Zealand; ${ }^{2}$ School of Physiotherapy, Wellington School of Medicine, University of Otago, Dunedin, New Zealand
}

\begin{abstract}
Background: The study was concerned with the secondary functional compromise persons with long-term paraplegia contend with, that has been shown to be associated with wheelchair use. Of particular interest was the relationship between the level of thoracic spinal cord injury (SCI) and factors predisposing to rotator cuff disorders (RCD), one of the most common upper extremity musculoskeletal complications linked with long-term SCI. Disorders of these peri-articular structures have previously been associated with impaired trunk postural control and abnormal muscle strength ratios in both able-bodied groups and in paraplegic athletes. Despite their neurologically intact shoulder joint musculature, high-level and low-level paraplegics have different degrees of trunk and pelvic stability available to them during activities of daily living (ADL). This fundamental functional anatomical difference between high-level and low-level paraplegics had not previously been related to the diagnosis of RCD in a non-athletic long-term paraplegic population.
\end{abstract}

Methods: A descriptive cross sectional study was undertaken to demonstrate the differences in the prevalence of clinically diagnosed RCD in a high-level $(n=22)$ and a low-level $(n=20)$ group of persons with long-term paraplegia. Any perceived differences were then related to the functional anatomical variations between the two groups. Inferences were made based on factors predisposing to RCD known to exist among both able-bodied and paraplegic athletes. The 42 subjects completed the Wheelchair User's Shoulder Pain Index (WUSPI) to establish the presence or absence of shoulder pain and the Musculoskeletal Function Assessment (MFA) instrument to determine differences in functional ability and perceived degree of difficulty within five categories of ADL. Each participant underwent a clinical examination using validated provocative clinical tests and isometric muscle strength ratio testing.

Results: There was a higher prevalence of RCD in the high-level group $(P=0.009)$ which correlated with decreased trunk control $(P=0.009)$. Differences in ability to perform functional tasks were not shown to be greater in the high-level group, although there was a higher degree of perceived difficulty in ADL. Muscle strength imbalances between the shoulder adductors and abductors were shown to be greater in the high-level group.

Conclusions: The results of this study suggest that the functional anatomical differences that exist within paraplegia contribute to the propensity of high-level paraplegic persons to suffer from RCD. These findings send a clear message to service-providers that greater acknowledgement of the differences in trunk postural control specific to the level of thoracic SCI is required. This may have implications for the prescription of wheelchairs, assistive devices and instruction for alternative ADL techniques to this specific population.

Spinal Cord (2000) 38, $748-753$

Keywords: paraplegia; rotator cuff disorders; postural control; trunk stability; muscle strength ratios

\section{Introduction}

Researchers are giving more attention to predicting the long-term implications of spinal cord injury (SCI), in particular to the long-term health, medical and

*Correspondence: KA Sinnott, Rehabilitation Teaching and Research Unit, Wellington School of Medicine, PO Box 7343, Wellington, New Zealand psychosocial outcomes and issues associated with both aging and the duration of injury. ${ }^{1-3}$ When improved survival is coupled with the fact that SCI typically occurs in early adulthood, it is clear that many SCI individuals are now likely to face several decades of living with the consequences of severe disability. It has only recently been acknowledged that SCI is not the 
static and unchanging condition that it was once thought to be. As spinally injured persons are surviving longer, a new set of problems has arisen as aging of the musculoskeletal system is superimposed on pre-existing disability. ${ }^{4}$

Although there are discrepancies in the reported incidence of shoulder joint complex problems, the literature suggests that they become more prevalent by 12 to 15 years post-SCI. ${ }^{5-10}$ It is widely acknowledged that the problems occurring in the shoulder joint complex of SCI persons are a consequence of the increased stresses imposed by the reliance on the upper extremities. ${ }^{4,8}$ However, little reference is made to the functional anatomical considerations associated with the weightbearing role the shoulder joint complex is forced to adopt, and even less to the significance of the postural adaptations associated with the level of SCI. Deterioration of the shoulder joint complex, particularly among paraplegic persons, has been linked with excessive and/or repetitive loading associated with wheelchair mobility, depression lift transfers and activities of daily living (ADL). ${ }^{4,6-10}$ Maintenance of the high level of independence attained is dependent on the integrity of these joints.

When comparing the results of studies previously undertaken it is difficult to draw conclusions since the samples vary in age, gender, duration post-SCI and the method of defining mobility or lesion level (within the two distinct groups of tetraplegia and paraplegia). The relationship between the functional anatomy of the paraplegic's neurologically intact upper limb and shoulder joint complex superimposed on either a stable or unstable trunk and pelvis and the predisposition to shoulder joint complex problems had not been specifically applied to the level of the thoracic SCI. Also, little has previously been made of the differences within paraplegia, despite evidence that differences do exist. $^{11,12}$ It is postulated that these are associated with the lack of trunk stabilisation due to the absence or impairment of abdominal and spinal muscle control that occurs in high-level paraplegia. Paraplegics with low-level SCI are able to partially support their body weight using functional abdominal and spinal muscle action. ${ }^{11}$ Although reference has been made to the functional limitations resulting from the absence of abdominal and spinal musculature associated with SCI above $\mathrm{T} 8$, rehabilitative procedures do not appear to be considerate of this functional anatomical consideration. ${ }^{11,12}$

There have been extensive investigations into the mechanics of wheelchair propulsion, depression lift transfers, weight-relief lifts and the effects of aging and duration of SCI. ${ }^{12}$ Specific to the current investigation are findings suggesting that a relative shoulder adduction (ADD) to abduction (ABD) strength and internal rotation (IR) to ABD weakness have a causative role in the development of shoulder problems including RCD in the athletic paraplegic population. ${ }^{6,13-14}$ Central to the current study is a report, by Powers et al, ${ }^{11}$ of a relationship between internal rotator muscle weakness and poor trunk stabilisation. These authors postulate that compromised trunk stability plays a significant role in shoulder torque. Their results showed a statistically significant isometric weakness of the glenohumeral joint $(\mathrm{GHJ})$ internal rotators in the high-level paraplegic. They postulate that the trunk stabilisers provide a more erect posture and better support of the shoulder joint complex, thereby facilitating greater maximal isometric efforts in those subjects with neurologically intact trunk stabilisers. More recently, differences in postural muscle responses in both highlevel and low-level paraplegic groups verify this association. $^{15}$

The specific objectives of this component of the investigation were to compare both the prevalence and functional implications of clinically diagnosed RCD between high-level and low-level persons with longterm paraplegia; and to identify associations between the presence or absence of trunk postural control and RCD, and the presence or absence of muscle strength ratio imbalances within each of the two groups specific to the level of thoracic SCI.

\section{Methods}

A cross-sectional study of the entire population of male SCI clients on record at the Spinal Injuries Unit (SIU), Burwood Hospital, Christchurch (referred to as Burwood SIU) who met the inclusion criteria was undertaken.

\section{Subjects}

The study was limited to male paraplegic persons, due to the availability of subjects, demographics and anthropometrical differences between male and female persons. A sample of 48 clients under review by the Burwood SIU, with a past history of traumatic SCI and self-reported shoulder problems was sourced. All male clients with a history of traumatic SCI at 10-24 years post-injury with complete spinal cord lesion $(n=56)$ were included in the target population and a history of shoulder pain was determined from the clinical records. Attempts to contact these subjects were made, resulting in a sample population of 42 participants $(85.7 \%$ response rate). The high-level group $(n=22)$ was defined by a T2 to T7 lesion level, the low-level group $(n=20)$ by T8 to T12 lesion level.

\section{Measures}

Simple descriptive information was used to ascertain the homogeneity of the two groups; including age, years post-SCI, bodyweight (excluding fat density) and depression lift transfers per day. Physical and lifestyle characteristics, vocational status and recreational involvement were included in the analysis, however, these results are not discussed in this paper. The 
clinical diagnosis of RCD (using the physical examination described by Maitland ${ }^{16}$ ) within each group was compared, along with the presence or absence of trunk postural control. This assessment included five critical tests and was used to gain a clinical impression of the integrity of the rotator cuff musculature.

The Wheelchair Users Shoulder Pain Index $^{17}$ (WUSPI) and the Musculoskeletal Function Assessment $^{18}$ (MFA) index were used as self-report questionnaires to determine the presence or absence of impairment with ADL. ${ }^{19}$ Language used within both the WUSPI and MFA required some adjustment for a New Zealand population. Five categories were used to determine any differences between the two groups. These were mobility, self-care, rest and sleep, recreation and leisure, and lifestyle adjustment, coping and adaptation. Two sets of data were formed from each of the five categories. This style allows for both a dichotomous yes/no response to the ability to perform a task (Question Ratings Scores) and the score from an ordinal scale $0-5$ used to determine the degree of difficulty (Category Ratings Scores). ${ }^{18}$

The physical examination included the five impingement syndrome diagnostic tests described by Burnham et $a l^{6}$ and substantiated by Gill and Magarey, ${ }^{20}$ Magarey et $a l,{ }^{21}$ and Magarey. ${ }^{22}$ These included tests to determine the presence of a painful arc in either ABD or elevation; a painful response to the impingement position; wasting of the supraspinatus or the infraspinatus fossae; pain on resisted ABD with either IR or external rotation (ER); and a positive response to an anterior or inferior instability test. Muscle strength testing was undertaken using a maximal isometric contraction against resistance using a pressure biofeedback cuff (Pressure Biofeedback Stabiliser, Chattanooga Pacific). Differences between the two groups in the strength ratios of $\mathrm{ABD}$ to $\mathrm{ADD}$, $\mathrm{ABD}$ to IR, and $\mathrm{ABD}$ to ER were determined.

\section{Procedures}

Ethical approval and informed subject consents were completed. The WUSPI and MFA were pre-tested by inpatient paraplegics at the Burwood SIU with reported shoulder problems associated with ADL and who did not fulfil the criteria to participate in this investigation. Prior to analysis the intra-rater, interrater reliability, specificity and face validity of the measures were tested in a Pilot Study undertaken with a sample of 10 persons with paraplegia in the inpatient facility at the Burwood SIU. The results were analyzed in view of the extensive analysis of the psychometric properties undertaken on the WUSPI ${ }^{23}$ and MFA. ${ }^{24}$ This pre-testing of the instruments also assisted with determining the functional relevance, language difficulties and completion time. ${ }^{12}$

Completion of the questionnaire preceded the physical examination and the investigator had no knowledge of each participant's response to the questionnaire. Clinical assessments were undertaken by an experienced physiotherapist, without prior knowledge of the diagnosis of the SCI consultant (taken from the clinical records). The critical tests were undertaken by the assessor and where possible repeated by a blinded research assistant. This was not achieved for seven persons (three high-level and four low-level) due to geographical difficulties.

The sitting posture of the participant was observed both in his wheelchair and seated on a low plinth or table without support. Posterior and lateral twodimensional photographs were taken to verify the contour of the thoracic kyphosis; the resting position of the head; the resting position of the scapulae; the position of the scapulae during an attempt to maintain sitting balance; the position of the scapulae during an attempt to correct sitting posture; and the position of the scapulae during a cough. The ability to maintain and correct two postures were observed and during the physical examination.

The critical tests used to clinically diagnose RCD were undertaken on both dominant and non-dominant shoulders. Isometric muscle tests were undertaken with the participant seated upright on a low plinth or table using the opposite hand to hold onto the front edge of the surface. The shoulder was in neutral with $90^{\circ}$ elbow flexion and neutral supination and pronation. Using the biofeedback cuff the maximum isometric reading of the best of three of $A B D, A D D, I R$ and $E R$ were recorded using a stable wooden platform vertically aligned to the subject for him to resist.

\section{Statistical analysis}

Descriptive statistics were used to describe, organise and summarise the data for each group for age, years post SCI, bodyweight and transfers per day. Appropriate non-parametric tests were used for non-normally distributed variables: Pearson Chi-square test was used to ascertain whether there were any differences across the two groups for the clinical diagnosis of RCD using critical tests; Kruskal-Wallis and the Mann-Whitney $U$ tests for both MFA scores and Levene's robust test of homogeneity of variances ${ }^{25}$ was used on the muscle strength ratio scores because of the abnormal distribution of the data. Inferences were made, where possible, against predisposing factors to RCD known to exist among both the general able-bodied population, and able-bodied and paraplegic athletes. The level of significance was set at 0.05 .

\section{Results}

There were no significant differences in baseline characteristics between the high-level and low-level groups. The low-level group performed a mean three transfers per day more than the high-level group (difference not significant), but mean age (44.7 vs 44.6 years), years post SCI (19.1 vs 18.9 years) and bodyweight $(80.9$ vs $79.5 \mathrm{~kg}$ ) were virtually identical. (Table 1). 


\section{Functional assessment instruments}

The WUSPI confirmed that all participants reported shoulder pain while performing ADL. There were no differences between the two groups in the ability to perform the tasks within the five categories of the MFA tested (Question Rating Scores). However, variation between the high-level and low-level groups in the perceived degree of difficulty associated with undertaking a functional task in the presence of shoulder pain, as determined using the Category Rating Scores, were evident for all of the MFA Category Rating Scores (Table 2).

\section{Clinical diagnosis of rotator cuff disorders}

Eighteen of the 22 high-level paraplegics $(81.8 \%)$ and eight of the 20 low-level paraplegics (40\%) were diagnosed with RCD (Pearson Chi-Square test, $(P=0.009)$. Fourteen of the high-level group had involvement of both shoulders and four the nondominant (Ndom) shoulder only (Table 3). Of the lowlevel group, four presented with both shoulders affected, and four with Ndom only. No individuals in either group presented with RCD affecting the dominant (dom) shoulder only.

\section{Postural control}

Fourteen of the high-level group $(63.6 \%)$ and four of the low-level group $(20 \%)$ presented with both an incorrectable thoracic kyphosis and incorrectable scapulae protraction. Four of the high-level group $(18.1 \%)$ and four of the low-level group (20\%) were

Table 1 Descriptive statistics of age, years post-spinal cord injury, bodyweight, and transfers per day of high-level $(n=22)$ and low-level $(n=20)$ groups

\begin{tabular}{llccc}
\hline Variable & Level & Mean & Median & $S D$ \\
\hline Age (years) & high & 44.6 & 43.5 & 10.4 \\
& low & 44.7 & 42.0 & 9.4 \\
Years post-SCI & high & 18.9 & 18.5 & 3.2 \\
& low & 19.1 & 20.0 & 2.7 \\
Bodyweight (kg) & high & 79.5 & 81.0 & 14.4 \\
& low & 80.9 & 85.0 & 12.0 \\
Transfers/day & high & 11.5 & 11.5 & 4.3 \\
& low & 14.5 & 14.5 & 6.9 \\
\hline
\end{tabular}

Table 2 Comparison of median category rating scores $(0=$ no problems; $5=$ extreme difficulty) between high-level $(n=22)$ and low-level $(n=20)$ groups

\begin{tabular}{lccc}
\hline Categories & High & Low & P value \\
\hline Mobility & 4.5 & 2.0 & 0.01 \\
Self-care & 4.0 & 1.0 & 0.02 \\
Rest and sleep & 5.0 & 1.0 & 0.002 \\
Recreation and leisure & 5.0 & 2.0 & 0.01 \\
Lifestyle changes & 5.0 & 1.5 & 0.01
\end{tabular}

able to correct only the increased scapulae protraction (Table 4).

\section{Relation between diagnosis of RCD and impaired postural control}

Sixteen of the high-level group $(72.7 \%)$ with both incorrectable postural habits were positively diagnosed with bilateral RCD as compared with only three of low-level participants $(15 \%)$. One of the low-level group with one impaired postural habit was diagnosed with RCD affecting the Ndom shoulder only. There were two high-level persons $(9 \%)$ presenting with neither clinically diagnosed RCD nor impaired postural control compared with eight in the low-level group $(40 \%)$. Overall, there was a significant relationship between any incorrectable posture and any diagnosis of RCD (Pearson Chi-Square test $=12.48$, $d f=3, P=0.006)$.

\section{Muscle strength ratios}

The only difference shown between high-level and lowlevel groups was for the ABD to ADD isometric muscle strength ratios on the non-dominant (Ndom) shoulder $(P=0.006)$

\section{Discussion}

There is general consensus that shoulder pain is commonly associated with wheelchair use and that the most frequent diagnoses are of a musculoskeletal origin. $^{12,26}$ Studies of shoulder joint complex problems and SCI have differentiated between tetraplegia (with some distinctions made between levels) and paraplegia but have generally not acknowledged the differences within paraplegia. ${ }^{12}$ The paucity of studies aimed

Table 3 Comparison of clinical diagnosis of rotator cuff disorders

\begin{tabular}{lccccc}
\hline & $\begin{array}{c}\text { High } \\
(\mathrm{n}=22)\end{array}$ & $\begin{array}{c}\text { Low } \\
(\mathrm{n}=20)\end{array}$ & $\begin{array}{c}\text { Total } \\
(\mathrm{n}=42)\end{array}$ & $\begin{array}{c}\mathrm{P} \\
\text { value }\end{array}$ & $\chi^{2}$ \\
\hline $\begin{array}{l}\text { Either shoulder } \\
\text { Non-dominant shoulder }\end{array}$ & 18 & 8 & 26 & 0.009 & 9.5 \\
$\quad$ only & 4 & 4 & 8 & & \\
$\begin{array}{l}\text { Dominant shoulder } \\
\quad \text { only }\end{array}$ & 0 & 0 & 0 & & \\
$\quad \begin{array}{l}\text { Neither shoulder } \\
\text { Both shoulders }\end{array}$ & 4 & 12 & 16 & & \\
\hline
\end{tabular}

Table 4 Comparison of incorrectable postures

\begin{tabular}{lccccc}
\hline & $\begin{array}{c}\text { High } \\
(\mathrm{n}=22)\end{array}$ & $\begin{array}{c}\text { Low } \\
(\mathrm{n}=20)\end{array}$ & $\begin{array}{c}\text { Total } \\
(\mathrm{n}=42)\end{array}$ & $\begin{array}{c}\mathrm{P} \\
\text { value }\end{array}$ & $\chi^{2}$ \\
\hline Either posture & 18 & 8 & 26 & 0.009 & 9.5 \\
Neither posture & 4 & 12 & 16 & & \\
Both postures & 14 & 4 & 18 & & \\
\hline
\end{tabular}


specifically at determining an association between trunk postural control and RCD provided the research question.

Results of the self-reported functional assessment instrument indicated that even in the presence of clinically diagnosed RCD, both groups (as expected) maintained a high level of functional independence. However, the degree to which the individual was functionally compromised was higher for the high-level group within each of the five categories tested. The differences in the degree to which individuals were functionally compromised while performing ADL tasks within the high-level group verifies that it is not sufficient to measure ability to perform functional tasks when attempting to ascertain functional status. ${ }^{19,27}$

It could be argued that the high-level group may have more difficulty with the functional tasks because of greater impairment associated with the lesion level. This was considered by the investigators but accounted for by determining whether the level of independence between the two groups was different. Both groups were involved in a wide range of sport and recreation activities with no obvious differences in participation between the two groups. The exception to this was in the presence of bilateral RCD three high-level subjects had changed, but not discontinued, their sporting activities due to bilateral shoulder pain.

Clinically diagnosed RCD was shown to be more prevalent in the high-level group and this correlated as expected, with greater evidence of impairment of both incorrectable habitual sitting postures amidst the highlevel group. However, because magnetic resonance imaging (MRI) or other imaging modality had not been available to confirm lesion level, anatomical variation of the innervation of abdominal and spinal musculature was not accounted for.

The difference between the two groups in shoulder ABD to ADD strength affecting the Ndom shoulder is of clinical interest because of the alleged relationship between RCD and muscle imbalances of the shoulder joint complex within the able-bodied athletic population. ${ }^{28-30}$ However, given that no other significant muscle imbalances could be demonstrated, this result may be due to chance. The results of Burnham et $a l^{6}$ and Powers et $a l^{11}$ showing more widespread significant muscle imbalances at the shoulder joint complex were not reproduced. The geographical distribution of the 42 participants throughout New Zealand precluded the use of isokinetic muscle testing.

\section{Limitations}

It would have been preferable to analyze a mid-level group (T6-8) separately. The observation and photographic verification of habitual postures was limited. It would have been useful to use a device known as a scapulocator ${ }^{31}$ to reliably measure position of the scapulae in three dimensions to determine whether the differences in postural control, shown to be significant in the sample population, could be reproduced more accurately in another sample. This would have allowed for the results to be applied more broadly within paraplegia. However, more recent studies by Seelen et $a l^{32,33}$ and Potten et al ${ }^{15}$ provide further evidence that postural control differences exist within paraplegia.

Functional expectations of paraplegics appear to be the same irrespective of the level of SCI; namely that manual wheelchair propulsion is usual and independent depression-lift transfers are a prerequisite for discharge following acute restorative rehabilitation. ${ }^{12}$ The level of SCI within the larger population referred to as 'paraplegia' is frequently not defined, other than within competitive disabled sports organisations where differences in functional ability are based on the level of SCI. ${ }^{34}$ Upper limb strengthening and stretching regimes are not necessarily cognisant of the functional anatomy of the shoulder joint complex and frequently complications associated with the role of the shoulder as the weightbearing 'ambulatory' structure do not appear to be acknowledged until pain and/or pathology exist. Furthermore, fashion-trends previously dictated the wheelchair design chosen, regardless of the presence or absence of effective sitting balance and neuromuscular control of the trunk stabilisers. The use of additional assistive devices to improve trunk postural control appear neither readily available nor acceptable to many paraplegic persons as they strive to maximise the capacity of intact motor function.

\section{Recommendations}

Individuals with SCI have historically demonstrated the ability to learn to modify their motor behaviour to best suit their physical capacity. ${ }^{26}$ It therefore seems only reasonable to suppose that functional expectations are based on realistic possibilities - if functional differences within paraplegia exist they need to be acknowledged. Modification of ADL techniques, wheelchair adaptations and the use of assistive devices may successfully override feelings of dependence if persons are educated appropriately during the early restorative phase.

Strategies may be as simple as ensuring that all paraplegic persons able to manoeuvre a manual wheelchair are provided with information on the functional anatomical limitations specific to their level of SCI including the implications of trunk stability and postural control mechanisms. Basic anatomy and joint mechanics could be easily described using readily accessible teaching tools.

Although speculative, it is suggested by the authors that the following strategies be implemented. Firstly, as a precaution against the development of $\mathrm{RCD}$, the paraplegic person with limited trunk control requires vigilance with suitable wheelchair back support, consideration of abdominal binders or similar adaptative devices for more vigorous wheeling activities, and conscientious care with upper limb strengthening and stretching regimes. It is beyond the 
scope of this paper to outline specific upper extremity strengthening and stretching regimes that acknowledge the lack of trunk control and pelvic stability in the paraplegic with compromised sitting postural control.

Management of the persons already presenting with evidence of RCD also provides sufficient material for a separate discussion. The key issues relate to improving trunk support, careful monitoring of wheeling as a form of 'exercise', and consideration of the integrity of the larger axio-humeral and axio-scapular muscles that provide secondary support to the rotator cuff and surrounding structures. Although controversial, it is also suggested that clinicians lower their threshold for consideration of powered wheelchair alternatives. Although not usual in New Zealand, early intermittent use of powered alternatives may be the single most effective measure for improving the life expectancy of the 'weight bearing' shoulder.

Obviously these interventions need to be tested and a randomised control trial would be the recommended research design. Successful pragmatic adaptation to the use of assistive technology will only occur in spinally injured persons if evidence-based practices shape the fundamentals of clinical management.

\section{Acknowledgements}

The study was generously funded by the New Zealand Spinal Trust and the New Zealand Returned Services' Association (NZRSA). Special thanks to Professor Alan Clarke, Mr Alan Bean, Dr Angelo Anthony, Mr Khalid Mohammed and Mrs Wendy Fulton at the Spinal Injuries Unit, Burwood Hospital, Christchurch, New Zealand for their combined enthusiasm, support and guidance. Many thanks to Dr Susan Mercer, Dept. of Anatomy and Structural Biology, for supervision of this project and assistance in the preparation of this paper, and $\mathrm{Dr}$ Laimonis Kavalieris, Dept. of Mathematics and Statistics, for statistical support; both from the University of Otago.

\section{References}

1 Gerhart KA et al. Long-term spinal cord injury: Functional changes over time. Arch Phys Med Rehabil 1993; 74: 1030-1034.

2 Pentland W, McColl MA, Rosenthal C. The effect of aging and duration of disability on long-term health outcomes following spinal cord injury. Paraplegia 1995; 33: $367-373$.

3 Whiteneck GG et al. Mortality, morbidity, and psychosocial outcomes of persons spinal cord injured more than 20 years ago. Paraplegia 1992; 30: 617-630.

4 Pentland W, Twomey L. The weightbearing upper extremity in women with long-term paraplegia. Paraplegia 1991; 29: 521 - 530.

5 Bayley J, Cochran T, Sledge C. The weightbearing shoulder. $J$ Bone Joint Surg 1987; 69A: 676-678.

6 Burnham R et al. Shoulder pain in wheelchair athletes. The role of muscle imbalance. Am J Sports Med 1993; 21: 238 -242.

7 Gellman H, Sie I, Waters R. Late complications of the weightbearing upper extremity in the paraplegic patient. Clin Orth Related Res 1988; 233: $132-135$.

8 Pentland W, Twomey L. Upper limb function in persons with long-term paraplegia and implications for independence: Part I. Paraplegia 1994; 32: $211-218$.
9 Pentland W, Twomey L. Upper limb function in persons with long-term paraplegia and implications for independence: Part II. Paraplegia 1994; 32: 219-224.

10 Sie I, Waters R, Adkins R, Gellman H. Upper extremity pain in the post rehabilitation spinal cord injured patient. Arch Phys Med Rehabil 1992; 73: 44-48.

11 Powers CM et al. Isometric torque in subjects with spinal cord injury. Arch Phys Med Rehabil 1994; 75: $761-765$.

12 Sinnott KA. The functional implications of rotator cuff disorders associated with chronic paraplegia; Master of Physiotherapy Thesis 1998, University of Otago, Dunedin.

13 Perry J, Gronley J, Newsam C, Reyes M, Mulroy S. Electromyographic analysis of the shoulder muscles during depression transfers in subjects with low-level paraplegia. Arch Phys Med Rehabil 1996; 77: 350-355.

14 Reyes ML et al. Electromyographic analysis of shoulder muscles of men with low-level paraplegia during a weight relief raise. Arch Phys Med Rehabil 1995; 76: 433-439.

15 Potten YJ, Seelen HA, Drukker J, Drost MR. Postural muscle responses in the spinal cord injured persons during forward reaching. Ergonomics 1999; 42: 1200-1215.

16 Maitland GD. Peripheral Manipulation, 3rd edn. Butterworth Heinemann: Oxford, 1991, pp. 129-138.

17 Curtis KA et al. Development of the wheelchair user's shoulder pain index (WUSPI). Paraplegia 1995; 33: 290-293.

18 Martin D et al. Development of a musculoskeletal extremity health status instrument: The musculoskeletal function assessment instrument (MFA). J Orthopaed Res 1996; 14: 173-181.

19 World Health Organisation. International Classification of Impairments, Activities and Participation (ICIDH-2 Beta 1 draft), a manual of classifications relating to the consequences of disease, Geneva: WHO, 1997.

20 Gill R, Magarey ME. Shoulder passive translation and passive rotation range of movement of competitive male tennis players. Proc MPAA, Perth 1993; 27-28.

21 Magarey ME, Hayes MG, Frick RA, Trott PH. The shoulder complex: A preliminary analysis of diagnostic agreement reached from a physiotherapy clinical examination and an arthroscopic evaluation. Proc MPAA Queensland 1995; $92-94$.

22 Magarey ME. The shoulder complex: A diagnostic dilemma and management challenge. Proc MPAA Melbourne 1997; 105-113.

23 Curtis KA et al. Reliability and validity of the wheelchair user's shoulder pain index (WUSPI). Paraplegia 1995; 33: 595-601.

24 Engelberg R et al. Musculoskeletal function assessment instrument: criterion and construct validity. J Orthopaed Res 1996; 14: $182-192$.

25 Kotz S, Johnson NL (eds). Encyclopedia of Statistical Sciences: Icing the tail - Limit Theorems, Vol 4. John Wiley \& Sons: New York, 1983, pp 608-609.

26 Allison GT. The ability to transfer in individuals with spinal cord injury. Critical reviews in physical and rehabilitation medicine 1997; 9: $131-150$

27 Keller R. Measuring outcomes. (Editorial) J Orthopaed Res 1996; 14: $171-172$

28 David G et al. EMG and strength correlates of selected shoulder muscles during rotations of the glenohumeral joint. Clin Biomech (Bristol, Avon) 2000; 15: 95-102.

29 Jobe FW, Pink M. Classification and treatment of shoulder dysfunction in the overhead athlete. JOSPT 1993; 18: 427-431.

30 Wilk KE, Arrigo CA, Andrews JR. Current concepts: The stabilising structures of the glenohumeral joint, JOSPT 1997; 25: $364-379$

31 Sobush DC et al. The Lennie test for measuring scapular position in healthy young adult females: A reliability and validity study. JOSPT 1996; 23: $39-50$.

32 Seelen HA et al. Development of new muscle synergies in postural control in spinal cord injured subjects. $J$ Electromyogr Kinesiol 1998; 8: 23-34.

33 Seelen HA et al. Postural motor programming in paraplegic patients during rehabilitation. Ergonomics 1998; 41: 302-316.

34 Christchurch Spinal Injuries Unit. Practice Guidelines: Spinal Cord Injuries, 2nd edn. 1996, Burwood International Spinal Trust. 